\title{
Publisher Correction: Fusion guide RNAs for orthogonal gene manipulation with Cas9 and Cpf1
}

Jiyeon Kweon', An-Hee Jang ${ }^{1}$, Da-eun Kim²,3, Jin Wook Yang ${ }^{1}$, Mijung Yoon', Ha Rim Shin ${ }^{1}$, Jin-Soo Kim²,3 \& Yongsub Kim (1) ${ }^{1}$

Correction to: Nature Communications https://doi.org/10.1038/s41467-017-01650-w, Article published online 23 November 2017

The originally published version of this Article contained an error in the spelling of the author Da-eun Kim, which was incorrectly given as Da-Eun Kim. Furthermore, in Figure 1a, the Cas9 protein was positioned incorrectly during typesetting. These errors have now been corrected in both the PDF and HTML versions of the Article.

Published online: 16 January 2018

(C) Open Access This article is licensed under a Creative Commons Attribution 4.0 International License, which permits use, sharing, adaptation, distribution and

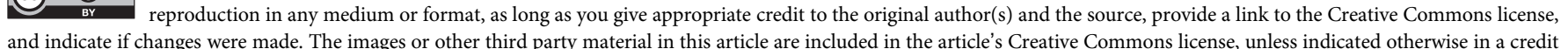

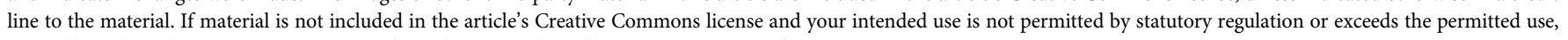
you will need to obtain permission directly from the copyright holder. To view a copy of this license, visit http://creativecommons.org/licenses/by/4.0/.

(C) The Author(s) 2018

\footnotetext{
${ }^{1}$ Department of Biomedical Science, University of Ulsan College of Medicine, Asan Medical Center, Seoul 05505, Republic of Korea. ${ }^{2}$ Department of Chemistry, Seoul National University, Seoul 08826, Republic of Korea. ${ }^{3}$ Center for Genome Engineering, Institute for Basic Science, Seoul 08826, Republic of Korea. Correspondence and requests for materials should be addressed to Y.K. (email: yongsub1.kim@gmail.com)
} 